\title{
O CONSUMO DE ÁlCOOL DURANTe O TRATAMENTO DA TUBERCulose: PERCEPÇÃO DOS PACIENTES
}

\section{Cleidiani Baptista da Silva $^{1}$; Rute dos Santos Lafaiete² ; Marilurde Donato $^{3}$}

O alcoolismo influencia no prognóstico da tuberculose (TB), e detectar seu uso, na Atenção Primária à Saúde, evita complicações. Objetivou-se descrever e analisar a percepção sobre o consumo de álcool no tratamento da TB, discutir as intervenções da equipe de saúde e da enfermagem. Trata-se de estudo descritivo, qualitativo, realizado no Rio de Janeiro, com 19 pacientes em tratamento da TB. Concluiu-se que os sujeitos percebem o consumo de álcool negativamente e os profissionais nem sempre orientam de forma contínua, mostrando que a enfermagem deve se inserir mais ativamente com ações educativas, de forma compreensiva. Recomenda-se a intervenção breve para a promoção da saúde e prevenção de danos à população.

Descritores: Tuberculose; Consumo de Bebidas Alcoólicas ; Atenção Primária à Saúde

\section{Alcohol use during tuberculosis treatment: Patients’ Perception}

Alcoholism affects the prognosis of tuberculosis (TB) treatment, and it is possible to avoid complications if alcohol use is detected at the Primary Health Care Center. The purpose of this study was to describe and analyze the perception of alcohol use during TB treatment, and discuss on the interventions by the health and nursing team. This descriptive, qualitative study was performed in Rio de Janeiro, with 19 patients following TB treatment. In conclusion, subjects see alcohol use as negative, and health professionals do not always provide continuous orientation; this shows that nursing should be more active by implementing comprehensive educational actions. Early intervention is recommended to promote health and avoid harms to the population.

Descriptors: Tuberculosis; Alcohol Drinking ; Primary Health Care

1-Enfermeira. E-mail: cleidiani.baptista@gmail.com

2-Enfermeira, Mestranda em Enfermagem, Escola de Enfermagem Anna Nery, Universidade Federal do Rio de Janeiro, RJ, Brasil. E-mail: rutelafaiete@bol.com.br

3-Enfermeira, Doutor em Enfermagem, Professor Adjunto, Escola de Enfermagem Anna Nery, Universidade Federal do Rio de Janeiro. E-mail: marilurdedonato1@terra.com.br

Endereço para Correpondência: 


\section{El CONSUMO DE ALCOHOL DURANTE EL TRATAMIENTO DE LA TUBERCULOSIS: PERCEPCIÓN DE LOS PACIENTES}

El alcoholismo influencia en la prognosis de la tuberculosis-TB, y detectar su uso en la Atención Primaria de Salud evita complicaciones. Se objetivó describir y analizar la percepción sobre el consumo de alcohol en el tratamiento de la TB, discutir las intervenciones del equipo de salud y de enfermería. Se trata de un estudio descriptivo, cualitativo, realizado en Rio de Janeiro con 19 pacientes en tratamiento de TB. Se concluyó en que los sujetos perciben negativamente el consumo de alcohol y los profesionales no siempre los orientan de forma continua; demostrando que la enfermería debe insertarse más activamente en acciones educativas de forma comprensiva. Se recomienda la intervención breve para la promoción de la salud y prevención de daños a la población.

Descriptores: Tuberculosis; Consumo de Bebidas Alcohólicas ; Atención Primaria de Salud. 


\section{Introdução}

A Organização Mundial da Saúde (OMS) estima que haja aproximadamente 2 bilhões de pessoas em todo o mundo consumidoras de bebidas alcoólicas e 76,3 milhões apresentam algum tipo de desordem como consequência do uso do álcool ${ }^{(1)}$. Em 2007, o Governo Federal lançou a Política Nacional sobre o Álcool (PNA), trazendo um conjunto de medidas para reduzir e prevenir os danos à saúde e à vida, apresentando princípios fundamentais à sustentação de estratégias, para o enfrentamento coletivo dos problemas relacionados ao consumo de álcool.

Uma das diretrizes da PNA consiste na detecção precoce do uso/abuso do álcool que é fundamental para prevenir problemas sociais e de saúde na comunidade, podendo ser realizado na Atenção Primária à Saúde (APS), pois essa se caracteriza por um conjunto de ações de saúde, no âmbito individual e coletivo, que atinge a maior parte da população. Esse nível de atenção é estratégico para minimizar os problemas dos usuários dos serviços de saúde, da família e também da comunidade, sendo propício à detecção precoce. Uma das dificuldades é que os profissionais têm capacitação insuficiente para essa detecção. A capacitação é essencial para o rastreamento de indivíduos com problemas relacionados ao consumo de álcool $^{(2)}$.

Implantar ações de prevenção e controle não deveria somente limitar-se ao uso/abuso de álcool, mas, também, abranger todos os problemas de saúde passíveis de prevenção, já que esse é um dos objetivos da APS. Tornase um paradigma, pois, ao invés de priorizar a promoção e prevenção, na prática, há predominância do enfoque curativo nos serviços de saúde ${ }^{(3)}$. Detectar problemas com o álcool, na APS, propicia medidas de intervenções e de controle para os usuários atendidos nos serviços.

$\mathrm{O}$ alcoolismo exerce influência sobre o prognóstico e o tratamento da tuberculose (TB), visto que há alta incidência de casos e de formas mais avançadas de TB pulmonar entre pacientes alcoolistas ${ }^{(4)}$. O problema deve ser tratado na comunidade e, também, mais considerado pela equipe de saúde que trabalha diretamente com doentes de TB, buscando encontrar meios precisos de identificar esses pacientes e oferecer tratamento concomitante ao uso ou abuso de álcool.

Osalcoolistasapresentaramprobabilidadequasequatro vezes maior de abandonar o tratamento ${ }^{(4)}$. A sensação de recuperação de saúde, o uso da bebida alcoólica ou drogas e o desconforto do tratamento - incluindo efeitos adversos ${ }^{(5)}$ - são motivos para o abandono do tratamento, segundo a percepção de alguns pacientes. Detectar o consumo de álcool, ou sua dependência, durante o tratamento da TB, é importante para evitar possíveis complicações ao usuário do Programa de Controle da Tuberculose - PCT.

Esta pesquisa torna-se relevante à medida que, ao conhecer a percepção dos usuários do Programa de Controle da Tuberculose, acerca das consequências do consumo de álcool, durante o tratamento da TB, permitirá que a equipe de saúde, particularmente a enfermagem, proponha formas de intervenção adequadas para a problemática do uso de álcool, durante o tratamento da TB. Saber as percepções dos doentes de TB, durante o tratamento, é importante para o desenvolvimento de medidas que possam auxiliar nesse período.

Os objetivos da pesquisa foram: descrever e analisar a percepção dos usuários do PCT acerca do consumo de álcool, em qualquer quantidade, durante o seu tratamento; discutir as intervenções da equipe de saúde e, em particular, a da enfermagem junto aos usuários do PCT.

\section{Metodologia}

O estudo é do tipo descritivo com abordagem qualitativa, realizado em um centro municipal de saúde, localizado no município do Rio de Janeiro, responsável pelo atendimento da população do entorno da Área Programática 1.0 (AP 1.0). A coleta de dados ocorreu no segundo semestre de 2008. Como sujeitos da pesquisa, foram entrevistadas 19 pessoas em tratamento, ou que já concluíram o tratamento.

Vale informar que a presente pesquisa foi aprovada pelo Comitê de Ética em Pesquisa da Escola de Enfermagem Anna Nery da Universidade Federal do Rio de Janeiro, em 25 de abril de 2007, sob Protocolo n 031-07.

Visando conseguir a melhor apreensão da realidade, foi escolhida a entrevista individual como instrumento para a coleta de dados, utilizado um roteiro semiestruturado, contendo perguntas abertas, para permitir que o entrevistado se expressasse livremente. Com o objetivo de caracterização dos sujeitos da pesquisa; foram coletados dados como: idade, sexo, estado civil, renda, uso de bebida alcoólica, outras doenças, os tempos de tratamento da TB, de abstinência ao uso de álcool e do consumo de álcool.

Para atender ao primeiro objetivo do estudo, foram levantados dados referentes à percepção dos sujeitos sobre as consequências do consumo de álcool, em qualquer quantidade, durante o tratamento de TB, constituindo-se, assim, a primeira categoria. Referentes ao segundo objetivo da pesquisa, foram estudadas as intervenções da equipe de saúde que resultaram na segunda categoria. Também foram levantadas as intervenções da enfermagem junto aos usuários de TB, emergindo uma terceira categoria. Os sujeitos da pesquisa foram identificados pela letra ' $E$ ', de entrevistado, e através do número correspondente, como exemplo: $\mathrm{E}_{1}, \mathrm{E}_{6}$.

A técnica de análise de conteúdo foi utilizada como referencial metodológico. Os resultados foram analisados em consonância com a literatura científica sobre os temas álcool e TB, através da leitura de artigos científicos em meio eletrônico e em livros. Utilizou-se, como marco teórico, a revisão não sistemática de Andrade, Villa e Pillon, $2005^{(4)}$, sobre a influência do alcoolismo no prognóstico e tratamento da TB.

\section{Análise e Discussão dos Resultados}

Dos 19 entrevistados, 15 são do sexo masculino e 13 pessoas solteiras. Quanto à faixa etária, quatro sujeitos se encontravam entre 18 e 27 anos, quatro entre 28 e 46 anos e 11 com 47 anos ou mais. Em relação à renda mensal, 
dois sujeitos não possuíam renda, cinco recebiam até um salário mínimo, nove sujeitos de 2 a 5 salários, um sujeito mais que 5 salários e dois não quiseram informar a renda mensal. Quanto ao fato de possuir outras doenças, onze relataram não estarem acometidos por qualquer outra doença além da TB, enquanto oito afirmaram possuir outras com morbidades como diabetes, hiperuricemia, hipertensão arterial, bronquite, pneumonia e hepatite como efeito adverso, provocado pelos medicamentos para o controle da TB.

Em relação ao consumo de álcool, 13 pessoas relataram que já fizeram uso de bebida alcoólica, 5 nunca o fizeram e 1 indivíduo não quis relatar a informação. Dos 13 sujeitos que afirmaram ter utilizado bebida alcoólica, fizeram uso em período entre 1 e 40 anos, em qualquer quantidade e frequência. Desses, somente um relatou não ter parado de consumir bebida alcoólica durante o tratamento de TB, um outro relatou haver deixado de consumir bebidas alcoólicas há mais de 10 anos, anteriormente ao diagnóstico da doença, e um terceiro sujeito não soube informar há quanto tempo não ingere. Os 10 dependentes restantes relataram abster-se desde o início do tratamento. A TB apresenta pior prognóstico em pacientes alcoolistas quando comparados com não alcoolistas, o uso é considerado condição clínica que causa agravamento do caso $^{(4)}$. Foram definidas as categorias discutidas a seguir.

Percepção dos usuários do PCT sobre o consumo de álcool, durante o tratamento da $T B$

Pode-se perceber a visão negativa que a associação entre remédio e álcool traz para os usuários e que a bebida piora o estado de saúde, trazendo complicações, além de influenciar na ação dos medicamentos. A TB, frequentemente, traz algumas mudanças na condição física dos portadores como fraqueza, falta de ar e emagrecimento. Com o início do tratamento, é comum que ocorra a melhora dos sintomas, mas, muitas vezes, os efeitos adversos dos remédios trazem desconforto para os doentes, e esse pode ser exacerbado se associado às bebidas alcoólicas.

Influi muita coisa. O álcool corta o efeito do remédio, não adianta tomar remédio e beber, alguns enchem a cara e voltam pior ainda durante a internação. Aumenta o desconforto, se você quer ficar tem que fazer tudo direito $\left(E_{3}\right)$.

$O$ álcool pode trazer complicações que a gente não imagina. Tomando um remédio forte deste, está procurando a morte... (E $\left.E_{2}\right)$.

Não combina, acho que se beber corta o efeito do remédio, senão acaba ficando pior $\left(E_{l}\right)$.

A associação dos remédios com o álcool é uma das variáveis que afeta o resultado terapêutico, acarretando interações medicamentosas e quanto maior o número de medicamentos que o paciente recebe maior é a possibilidade da ocorrência. É evento clínico em que os efeitos de um fármaco são alterados pela presença de outro, alimentação, bebida ou algum agente químico ambiental ${ }^{(6)}$.

Os pacientes percebem que há desconforto ao utilizar as medicações para tratamento da TB e que, ao misturar com o álcool, há ampliação desse incômodo. Os efeitos colaterais causam desconforto de forma sintomática, persistindo ocasionalmente durante a terapia. O gerenciamento inadequado desses efeitos contribui para o tratamento irregular e à não adesão(7)

Não se pode misturar doença com bebida. Devo cumprir o tempo certo porque sei que faz mal para mim. Eu já sabia que quando se toma remédio muito forte não pode beber nem fumar $\left(E_{4}\right)$.

$O$ efeito do remédio não faz. Conheço um amigo que faz tratamento, mas continua bebendo. Ele diz que se beber morre, e se não beber morre também. Tem que parar com tudo porque faz mal $\left(E_{5}\right)$.

Corta a eficiência da medicação. Não importa a quantidade. Éruim $\left(\mathrm{E}_{6}\right)$.

Para totalmente o efeito do remédio $\left(\mathrm{E}_{7}\right)$.

Não deve acontecer porque fui orientado que pode ocorrer lesões no fígado e é outro risco para a saúde. Se já está se tratando não se deve arrumar outro problema $\left(\mathrm{E}_{8}\right)$.

A isoniazida, especificamente, misturada com álcool, e rifampicina (indutora da atividade do citocromo hepático P450) aumentam a incidência de hepatite. Ela ocorre frequentemente em doentes HIV e alcoolistas ${ }^{(8)}$. O doente percebe o risco que pode ocorrer no fígado pelo uso de bebida alcoólica, concomitante ao tratamento de TB. Toda orientação, em relação ao uso das medicações por esse tipo de paciente, deve enfatizar que a ingestão de bebidas alcoólicas aumenta o risco de efeitos colaterais, cabendo aos profissionais a observação contínua e sistematizada desses pacientes, durante o tratamento da TB, no sentido de minimizar e detectar precocemente possíveis efeitos dessa interação ${ }^{(4)}$. Em outras falas, evidencia-se a percepção sobre o uso do álcool como interferência negativa para o tratamento da TB:

Eu acho que a pessoa não deve beber nada... penso que durante o tratamento deve ser assim ( $\left.E_{0}\right)$.

Quem utiliza não quer se tratar, ou faz o tratamento ou utiliza o álcool ( $\left.E_{10}\right)$.

$E$ burrice beber porque se sabe que faz mal e prejudica a si mesmo $\left(E_{11}\right)$.

O consumo joga fora a oportunidade de se tratar, de melhorar o mais rápido possível. Prejudica a saúde $\left(E_{12}\right)$.

Olha, o consumo de álcool... eu até acho que não faz bem pra ninguém... Não é vantagem não... $\left(E_{13}\right)$.

Todas as falas refletem a visão do mau prognóstico ao tratamento de TB com o uso do álcool. Os prejuízos que trazem ao próprio portador da TB não compensam seu consumo, podendo gerar atrasos para o tratamento já que o esquema básico é de curta duração, devendo o paciente ser curado em seis meses. Alcoolistas possuem tempo superior a seis meses, que é explicado pelo fato de serem encontradas maiores porcentagens de abandono e absenteísmo entre essa clientela, determinando maior tempo de inclusão no tratamento. Assim, os pacientes podem se tornar pacientes crônicos, tanto da doença quanto do serviço ${ }^{(4,9)}$. Em outras falas, os sujeitos relataram ter consumido bebida alcoólica durante a terapia, indicando o uso consciente do álcool, mesmo sabendo que poderia trazer prejuízos à saúde. Um dos sujeitos, sabendo que o consumo traz malefícios, decidiu interromper o esquema terapêutico e retomar o consumo de álcool. Tão logo decidiu retornar ao tratamento, parou novamente o consumo de álcool, demonstrando a influência do álcool em seu prognóstico. 
Não pode porque faz mal para saúde. Eu ficaria muito ruim se continuasse bebendo. Eu parei por conta própria (...) comecei o tratamento, parei e voltei a usar cerveja e vinho. Parei por uma semana porque hoje estou voltando para o tratamento. Eu ficaria muito ruim se continuasse bebendo $\left(E_{14}\right)$.

É errado, eu até consumi, mas eu sei que é errado $\left(E_{15}\right)$.

Outro sujeito, apesar de ter consumido bebida alcoólica, não relatou ter interrompido o tratamento para beber, mesmo sabendo dos prejuízos. A percepção de se ter um problema com alguma substância psicoativa pressupõe que ocorra, com o usuário, mudança no modo de avaliar algunsfenômenos. Talmudançanãoaltera,necessariamente, o prognóstico da situação - pois é frequente desejar e não conseguir mudanças em um padrão alterado de uso de substâncias ${ }^{(10)}$. Um dos sujeitos compreende que consumir pequena quantidade de bebida não traz prejuízos ao longo do tratamento. Sua percepção sobre o uso e a quantidade de álcool poderia ser influenciada a partir de orientações e acompanhamentos constantes, principalmente por esse usuário ter tal entendimento. Ocorre maior cooperação dos usuários em relação ao tratamento quando esses são submetidos a maior vigilância ${ }^{(4)}$.

Destaca-se que os profissionais de saúde deverão exercer essa "vigilância" de modo que considerem os aspectos socioculturais do consumo do álcool, identificando os comportamentos de risco e não reprovando a conduta dos pacientes. Esse ato aumentará a confiança entre o usuário do serviço de saúde e o profissional, proporcionando processo terapêutico dialógico.

Não pode. Um copinho bem pequeno não faz mal. Mas as pessoas que bebem muito não podem se cuidar, o meu problema não é bebida $\left(\mathrm{E}_{16}\right)$.

As intervenções da equipe de saúde sobre o consumo de álcool, durante o tratamento da TB

No contexto atual, o pensamento dos profissionais de saúde está voltado a considerar como conhecimento tudo o que foi cientificamente comprovado e o que a literatura afirma ser verdadeiro, muitas vezes desprezando ou se opondo ao senso comum. A partir da articulação das formas de conhecimento, pode-se desenvolver compreensão satisfatória do processo de promoção da saúde, mediado pela educação em saúde ${ }^{(11)}$. Abaixo, um sujeito expressa sua opinião sobre o consumo de álcool e sobre o beber social, aparentemente baseado no senso comum:

Acho que é prejudicial. Já que o tratamento não pode, não pode. Ninguém consegue beber 1 copo só. Eu não conseguia beber 1 só copo. Não existe beber socialmente $\left(\mathrm{E}_{18}\right)$.

Através de um estudo ${ }^{(12)}$, verificou que, de 34 pacientes portadores de doença grave do fígado, causada pelo álcool, apenas $9 \%$ deles tinham dependência da bebida, ou seja, muitos dos pacientes que se apresentam com doença hepática, causada pelo álcool, eram pessoas que bebem apenas socialmente. A fronteira entre o uso e dependência ao álcool é estreita, já que a sociedade insere a bebida em todas as comemorações e atividades de lazer. É questionada a existência do beber social e, no contexto do tratamento para a TB, o beber, em qualquer quantidade, é prejudicial.

Orientaram que não pode beber, e outras orientações. A médica que enfatizou sobre o uso da bebida alcoólica durante o tratamento da $T B\left(\mathrm{E}_{11}\right)$.

A orientação principalmente dos médicos é importante (...) Porque contribui para o tratamento adequado $\left(\mathrm{E}_{19}\right)$.

Acerca do conhecimento dos sujeitos sobre o consumo de álcool, durante o tratamento, verificou-se, aqui, que $\mathrm{E}_{8}$, citada anteriormente, e $\mathrm{E}_{11}$ e $\mathrm{E}_{19}$ relataram que receberam orientações de um profissional de saúde. Esse fato mostra a importância das orientações de um profissional de saúde nas consultas ou nos retornos frequentes para a tomada da medicação. As orientações devem abordar os efeitos colaterais e, ainda, que a ingestão de bebidas alcoólicas aumenta o risco para o desenvolvimento dos efeitos, dentre outros cuidados com a saúde. Demonstrou-se que suas percepções não são baseadas somente no senso comum, mas em orientações consistentes sobre o uso do álcool durante o tratamento.

Já em outras falas, mostram que os profissionais de saúde estão prestando assistência no controle da TB, contudo, não orientam quanto à exposição a fatores de risco que podem influenciar no tratamento. Há de se salientar que tanto a prática da enfermagem como a prática médica devem emergir, alternadas e direcionadas, de acordo com as necessidades de respostas aos problemas de saúde da população. As orientações devem permear não só o tratamento da doença, mas, também, os seus aspectos fisiológicos, alimentação e até mesmo os cuidados com a própria família. Percebe-se que a falta de conhecimento do paciente, a respeito da $\mathrm{TB}$, provoca situações nas quais fica comprometida a segurança de sua saúde, dificultando o tratamento.

Não foi (...) eu só venho e tomo o remédio e não falam $\operatorname{nada}\left(\mathrm{E}_{1}\right)$.

Não teve orientações, nem do médico e nem do enfermeiro $\left(\mathrm{E}_{5}\right)$.

Nota-se que alguns sujeitos não foram orientados durante as consultas médicas e de enfermagem. Outros sujeitos, apesar da falta de orientação, sentiram necessidade de pesquisar ou refletir, a partir de problemas do seu cotidiano, sobre o consumo de álcool:

Ninguém me orientou e caí na real com minha própria consciência $\left(\mathrm{E}_{7}\right)$.

Não houve orientação. Eu sei que devo cuidar da minha saúde e bebida não ajuda $\left(\mathrm{E}_{8}\right)$.

Os profissionais da atenção primária à saúde devem ser capazes de identificar e aplicar condutas nos pacientes cujo consumo de álcool esteja trazendo riscos para a sua saúde e para a cura da TB. É ideal que os profissionais busquem estratégias para viabilizar a comunicação entre profissionais da saúde e as comunidades envolvidas nas ações de saúde, para que as ações de autocuidado não sejam tomadas intuitivamente, permeadas pelo senso comum, sendo necessário que se estabeleça vínculo entre os profissionais e o cliente, para que o mesmo se sinta confortável para perguntar e exprimir suas opiniões e queixas.

A afirmação abaixo corrobora as orientações dos 
profissionais de saúde, mostrando que contribuíram positivamente no decorrer do tratamento.

Ajudou muito... para dar uma acalmada... para $\operatorname{parar}\left(\mathrm{E}_{4}\right)$.

As enfermeiras e médicos falam mesmo, e não discriminam $\left(\mathrm{E}_{18}\right)$.

As falas acima exemplificam quando a equipe multiprofissional realiza articulação de saberes, estabelecendo relação de diálogo e proximidade, pois o usuário se sentiu à vontade e tranquilo para buscar cumplicidade e confiança nos profissionais. Houve um discurso para que o cuidado fosse de qualidade, livre de preconceitos e atitudes discriminatórias, já a doença ainda possui caráter excludente e discriminatório. Indivíduos com TB sofrem com o aparecimento da doença, não só pelas manifestações clínicas, mas, também, pela possibilidade de vivenciar preconceitos. É muito importante que o atendimento seja livre de preconceitos e julgamento de valores. A divulgação do que é a doença, seus cuidados e tratamentos, através da mídia, são estratégias que podem trazer resultados mais efetivos para o viver mais saudável com a TB.

Durante as entrevistas, emergiram falas sugestivas para o serviço em questão, com a intenção de melhoria das orientações fornecidas pelos profissionais de saúde, demonstrando que houve interesse em relação ao aprendizado. Em uma fala também se argumentou o fato de não se receber mais orientações sobre a bebida e suas complicações, de forma mais detalhada.

Eles poderiam explicar bem explicado. Eles falam que não pode beber porque passa mal, mas não explica mais nada $\left(\mathrm{E}_{16}\right)$.

A educação em saúde, no Brasil, instituiu-se no âmbito da saúde pública, orientando novas práticas. Dentre essas, duas dimensões se destacam e persistem atualmente. A primeira envolve a aprendizagem sobre as doenças, prevenção, seus efeitos e como restabelecer a saúde. A outra tendência, caracterizada como promoção da saúde pela OMS, inclui os fatores sociais que interferem, abordando os caminhos pelos quais diferentes estados de saúde e bem-estar são construídos socialmente ${ }^{(13)}$.

As intervenções de enfermagem sobre o consumo de álcool, durante o tratamento de TB

Nas falas abaixo, verifica-se que a orientação médica foi dada em alguns atendimentos e não foi dada concomitante às orientações de enfermagem.

$O$ médico fez a orientação. A enfermagem não orientou. Seria útil se reforçassem o assunto apesar do médico já ter falado sobre isso $\left(\mathrm{E}_{3}\right)$.

Quem me falou foi o médico, e eu já sabia. A enfermeira não falou nada $\left(\mathrm{E}_{6}\right)$.

A médica orientou. Ninguém da enfermagem falou comigo $\left(\mathrm{E}_{9}\right)$.

Há duas noções que recobrem a ideia de equipe: uma como agrupamento de agentes e outra como integração de trabalhos. Nesse sentido, a comunicação entre os profissionais seria o denominador comum do trabalho em conjunto, que decorre da relação recíproca entre trabalho e interação ${ }^{(14)}$. A equipe de saúde deve interagir de forma contínua e integral, a fim de complementar o tratamento. De forma mais específica, o cuidado de enfermagem deve ser feito com empatia, incluindo atividades terapêuticas.

No primeiro atendimento, o enfermeiro pode identificar problemas associados ao consumo de álcool, estar atento às queixas do paciente, identificar padrões de consumo ao longo da história do paciente, na busca da caracterização do uso nocivo ou dependência ${ }^{(15)}$.

As informações foram dadas pela equipe de enfermagem $e$ ajuda para entender como o álcool atua no tratamento $\left(\mathrm{E}_{2}\right)$.

Ajudou muito. Falou da chuva, friagem e da bebida. Perguntou se bebia e falou para parar com tudo $\left(\mathrm{E}_{10}\right)$.

Todo mundo sabe que álcool e antibióticos não combinam. Orientaram sobre isso e diversas coisas $\left(\mathrm{E}_{12}\right)$.

Quanto às orientações de enfermagem, os usuários acima foram orientados e estimulados a persistirem no tratamento. Os relatos demonstram que, muitas vezes, a orientação é dada com o intuito da educação em saúde, porém, de forma imperativa. Ainda hoje, grande parte dos profissionais de saúde desenvolvem processo educativo com a finalidade linear de "corrigir" ou "superar" o conhecimento do outro ${ }^{(16)}$. Isso dá a ideia de que "o outro" possui uma deficiência. Verifica-se, então, que a orientação foi dada com o sentido de repressão, de suprimir um comportamento considerado pela equipe de enfermagem como ruim. Sendo asim, pode-se inferir que o processo de promoção e manutenção da saúde também se constitui em processo que envolve opiniões mútuas.

A enfermagem contribui falando que não pode faltar, não pode beber $\left(\mathrm{E}_{13}\right)$.

A enfermagem contribui com o incentivo psicológico para continuar o tratamento. Orientaram em relação à bebida, hábitos alimentares, atividades fisica e outras coisas $\left(\mathrm{E}_{15}\right)$.

As enfermeiras e médicos falam mesmo, e não discriminam $\left(\mathrm{E}_{18}\right)$.

A afirmativa de $\mathrm{E}_{15}$ mostra, em outras palavras, que os profissionais de saúde buscam outros referenciais além dos biológicos, pois se reconhece que as ações necessárias para a adesão a tratamentos e cuidados mais prolongados estão profundamente relacionadas à cultura, ou seja, com os estilos de vida, hábitos, rotinas e rituais na vida das pessoas. Sendo imprescindível que a postura não seja dominadora e excludente, mas, sim, compreensiva e de parceria, durante o tratamento. As orientações de enfermagem são importantes, pois são profissionais que estão diretamente envolvidos com o cuidado e com a gerência de recursos, além de serem intermediários entre a família, o doente e a equipe de saúde.

As orientações individuais ou em grupo possibilitam que habilidades pessoais sejam desenvolvidas, buscando a capacitação das pessoas através das experiências vividas para a obtenção do autocuidado. Em outras falas, há a sugestão de palestras, de sala de espera e de orientação coletiva. A partir disso, fica evidente a importância da atuação da enfermagem para a realização de orientações, não só feitas 
individualmente, mas também de forma coletiva.

Se tivesse palestras, grupo de sala ou espera, para passar a informação. A informação vale muito $\left(\mathrm{E}_{14}\right)$.

Deram-me orientação. Seria legal a realização de grupos de orientação com os pacientes $\left(\mathrm{E}_{17}\right)$.

O enfermeiro tem papel fundamental, faz-se necessário empregar a estratégia do ato de educar para a obtenção da saúde. Nessa abordagem, o profissional atua como facilitador, intermediário, negociador entre as necessidades daqueles que são cuidados e os diferentes sistemas da sociedade. É importante lembrar que a ação promocional/ educativa é um processo que se realiza a médio e longo prazo, com impactos sociais não imediatos ${ }^{(17)}$.

A educação em saúde deve ser realizada a partir de troca de conhecimentos com a população que se pretende ajudar, levando-a a desenvolver consciência crítica. Dessa interação do conhecimento da comunidade com o profissional da área da saúde poderá emergir uma terceira forma de saber eficiente e eficaz, por surgir da reflexão e das experiências individuais e coletivas ${ }^{(13)}$.

\section{Considerações Finais}

Os usuários do PCT compreendem, de forma negativa, o consumo de álcool durante o tratamento de TB, ou seja, a percepção é que o consumo de álcool traz malefícios, em qualquer quantidade, durante o tratamento. Para os sujeitos entrevistados, o consumo de bebida alcoólica, concomitante ao tratamento de TB, funciona como exacerbador do desconforto causado pela terapia medicamentosa.

Percebeu-se que tanto os profissionais da equipe de enfermagem como os médicos, que prestam a assistência no controle da $\mathrm{TB}$, nem sempre orientam quanto à exposição dos fatores de risco que influenciam no tratamento. Devese viabilizar a comunicação entre profissionais da saúde e entre os usuários do serviço para o desenvolvimento das ações de saúde, principalmente para que não sejam intuitivamente permeadas pelo senso comum.

Acerca das orientações de enfermagem, alguns usuários relataram que foram orientados e estimulados. A enfermagem, por ter atuação importante, deve se inserir mais ativamente através de palestras, sala de espera, orientação coletiva ou individual com postura compreensiva e de parceria, durante o tratamento.

Recomenda-se a intervenção breve, por meio da técnica de aconselhamento, para esse contexto, como forma de atendimento, concomitante ao tratamento da TB, no intuito de reduzir possíveis danos acarretados pelo uso do álcool, em qualquer quantidade, principalmente durante o tratamento, ou seja, de forma articulada a práticas terapêuticas, preventivas, educativas, de acordo com a Portaria n².197/GM, de 14 de outubro de $2004^{(18)}$.

Rastrear o uso ou o abuso do álcool, durante o tratamento da TB, a partir das percepções dos usuários do PCT, contribui significativamente para a promoção da saúde e prevenção de agravos à população, no contexto da APS.

\section{Referências}

1. Segatto ML, Pinsky I, Laranjeira R, Rezende FF, Vilela TR. Triagem e intervenção breve em pacientes alcoolizados atendidos na emergência: perspectivas e desafios. Cad Saúde Pública. jun 2007;23(8):1753-62.

2. Ronzani TM, Mota DCB, Souza ICW. Prevenção do uso de álcool na atenção primária em municípios do estado de Minas Gerais. Rev Saúde Pública. 2009;43(supl 1):51-61.

3. Ronzani TM, Ribeiro MS, Amaral MB, Formigoni MLOS. Implantação de rotinas de rastreamento do uso de risco de álcool e de uma intervenção breve na atenção primária à saúde: dificuldades a serem superadas. Cad Saúde Pública. 2005;21(3):852-61.

4. Andrade RLP, Villa TCS, Pillon SC. A influência do alcoolismo no prognóstico e tratamento da tuberculose. SMAD, Rev Eletrônica Saúde Mental Álcool Drog. (Ed. port.) [internet]. fev 2005;1(1). [acesso 12 abril 2010]. Disponível em: http://www2.eerp.usp.br/resmad/ verArtigo_port.php?idioma $=$ portugues $\&$ ano $=2005 \&$ volu $\mathrm{me}=1 \&$ numero $=1 \& \mathrm{id}=44$

5. Paixão LM, Gontijo, ED. Perfil de casos de tuberculose notificados e fatores associados ao abandono. Belo Horizonte, MG. Rev Saúde Pública. 2007;41(2):205-13.

6. Secoli SR. Interações medicamentosas: fundamentos para a prática clínica da enfermagem. Rev Esc Enferm USP. mar 2001;35(1):28-34.

7. World Health Organization. What is DOTS? A guide to understanding the WHO recommended TB Control Strategy known as DOTS. Geneva; 1999. WHO/CDS/ CPC/TB/99.270.

8. World Health Organization. Efeitos secundários dos medicametos anti TB/HIV: Manual clinico. Geneva; 2004. p. $135-43$.

9. Vendramini SHF. O tratamento supervisionado no controle da tuberculose em Ribeirão Preto sob a percepção do doente. [dissertação]. Ribeirão Preto (SP): Escola de Enfermagem de Ribeirão Preto/USP; 2001.

10. Fontanella BJB, Mello GA, Demarzo MMP, Turato ER. Percepção da síndrome de dependência por pacientes em tratamento. J Bras Psiquiatr. 2008;57(3):196-202.

11. Rios ERG, Franchi KMB; Silva RM, Amorim RF, Costa NC. Senso comum, ciência e filosofia: elo dos saberes necessários à promoção da saúde. Ciênc Saúde Colet. 2007;12(2): 501-9.

12. OBID - Observatório Brasileiro de Informações Sobre Drogas (BR). Beber socialmente pode causar cirrose 2006. [acesso 12 abril 2010]. Disponível em: http:/www.obid. senad.gov.br/portais/OBID/conteudo/web/noticia/ler_ noticia.php?id_noticia $=7258$.

13. Schall V. Stuchiner M. Educação em saúde: novas perspectivas. Rio de Janeiro 1999. Cad Saúde Pública 1999;2:4-5.

14. Peduzzi M. Equipe multiprofissional de saúde: conceito e tipologia. Rev Saúde Pública. fev 2001;35(1):103-9.

15.Pillon SC, Lius MAV. Modelos explicativos para o uso de álcool e drogas e a prática da enfermagem. Rev. LatinoAm. Enfermagem. ago 2004;12(4):676-82. 
16. Langdon EJ. Cultura e processos de saúde e doença. In: Anais do 1o Seminário sobre Cultura, Saúde e Doença; 1316 out 2003; Londrina, Brasil. Londrina (PR): VIGISUS/ FNS/MS; 2003. p. 91-107.

17. Silva SÉD, Vasconcelos EV, Padilha MICS. A educação em saúde como uma estratégia para enfermagem na prevenção do alcoolismo. Esc Anna Nery. dez 2007;11(4):699-705.

18. Ministério da Saúde (BR). Portaria GM n²197, de 14 de outubro de 2004. Redefine e amplia a atenção integral para usuários de álcool e outras drogas, no âmbito do Sistema Único de Saúde - SUS, e dá outras providências. [acesso 12 de abril 2010]. Disponível em http://dtr2001.saude.gov.br/ sas/PORTARIAS/Port2004/GM/GM-2197.htm.

\section{Como citar este artigo:}

Silva CB, Lafaiete RS, Donato M. O consumo de álcool durante o tratamento da tuberculose: percepção dos pacientes. SMAD, Rev. Eletrônica Saúde Mental Álcool Drog. (Ed. port.). jan-abr 2011 [acesso: nível em:

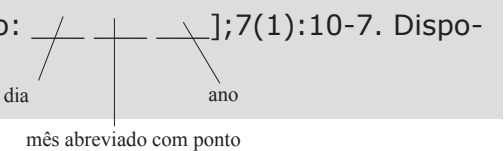

